\title{
Deconditioning as main mechanism of impaired exercise response in COVID-19 survivors
}

\section{Copyright (CThe authors 2021 \\ This version is distributed under the terms of the Creative Commons Attribution Non-Commercial Licence 4.0. For commercial reproduction rights and permissions contact permissions@ersnet.org}

This article has an editorial commentary: https://doi.org/10.1183/ 13993003.01763-2021

Received: 14 Jan 2021 Accepted: 17 April 2021

\section{Ә@®®}

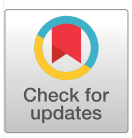

To the Editor:

SARS-CoV-2 (severe acute respiratory syndrome coronavirus 2) and the related coronavirus disease 2019 (COVID-19) hit Europe in February 2020 [1], raising issues on acute phase management and, later on, the management of its long-term sequelae. Cardiopulmonary exercise testing (CPET), which is the gold standard for the evaluation of exercise capacity, is included in the list of examinations of the European Respiratory Society/American Thoracic Society task force for the follow-up of COVID-19 patients [2]. However, it is not performed in every clinical centre, as it requires specific technical skills. The objective of this observational, prospective study was to evaluate the sequelae of COVID-19 by assessing exercise performance during incremental CPET.

COVID-19 patients who recovered from the acute phase were enrolled from the Registry for COVID-19 Emergency (RE.COV.ER), funded by the University of Milan, Italy. The study was approved by Milan Area 1 ethics committee (2020/ST/407). Written informed consent was obtained from each participant. Patients admitted between February and April 2020 and followed-up at the post-COVID-19 outpatient service at San Paolo Hospital (Milan, Italy), were invited to undergo CPET (May to August 2020). Inclusion criteria were: 1 ) age >18 years; and 2) molecular (RT-PCR) diagnosis of SARS-CoV-2 infection [3]. Exclusion criteria were the absence of signed informed consent, an acute respiratory exacerbation in the 4 weeks before enrolment, and the presence of medical conditions contraindicating CPET (acute or unstable cardio-respiratory conditions, osteo-muscular impairment that could compromise exercise performance) [4]. Information on past medical history, smoking status and COVID-19 therapies were collected. Dyspnoea sensation was assessed using the Italian version of the modified Medical Research Council dyspnoea scale (mMRC). SARS-CoV-2-related pneumonia diagnosis was based on specific radiological chest findings (radiographs: multifocal peripheral lung ground glass opacities and/or consolidations, monolateral or bilateral; computed tomography (CT): bilateral lung infiltrates, ground-glass opacities, consolidation, crazy paving pattern, air bronchogram signs and intralobular septal thickening). Patients underwent spirometry and diffusing lung capacity for carbon monoxide $\left(D_{\text {LCO }}\right)$ test evaluated by the single breath technique. Symptom-limited, incremental, exercise testing was performed on an electronically braked cycle ergometer using the Vmax Spectra Cardiopulmonary Exercise Testing System (SensorMedics, Yorba Linda, CA, USA) [4]. The rate of work rate increment $\left(\mathrm{W} \cdot \mathrm{min}^{-1}\right.$ ) was identified on an individual basis according to expected exercise tolerance and resting functional data. Measured and computed CPET variables were recorded [5]. Breathing reserve is $\left(1-\left(\right.\right.$ peak ventilation $\left.\left./\left(\mathrm{FEV}_{1} \times 35\right)\right)\right) \times 100$, where $\mathrm{FEV}_{1}$ is forced expiratory volume in $1 \mathrm{~s}$. Heart rate reserve (HRR) is (1-(peak heart rate/(220 -age)) $) \times 100$.

Chest CT signs were evaluated by a radiologist and a respiratory physician during the follow-up. Two CT scores were used to show the magnitude of the residual involvement: the CT severity score and the visual percentage of residual parenchymal involvement $[6,7]$.

Differences between patients with a preserved (peak oxygen consumption $\left(V^{\prime} \mathrm{O}_{2}\right) \geqslant 85 \%$ predicted [4]) and those with a reduced exercise capacity in terms of resting pulmonary function tests, ventilatory exercise response and imaging were computed. Based on early data on $D_{\text {LCO }}$ from COVID-19 patients [8], a sample size of at least 30 patients per group would be sufficient to detect a difference of $10 \%$ of predicted

Shareable abstract (@ERSpublications)

CPET reveals only a mild impairment of exercise capacity, with preserved ventilatory and gas exchange response at 3 months follow-up in COVID-19 survivors, due to deconditioning https://bit.ly/3sl8e0Y

Cite this article as: Rinaldo RF, Mondoni M, Parazzini EM, et al. Deconditioning as main mechanism of impaired exercise response in COVID-19 survivors. Eur Respir J 2021; 58: 2100870 [DOI: 10.1183/ 13993003.00870-2021]. 
$D_{\text {LCO }}$ (a minimal clinically relevant difference in respiratory diseases) between them (statistical power of $80 \%$, alpha error of $5 \%$ ). Student's t- or Mann-Whitney tests were computed to assess statistical differences for normal or non-normal quantitative variables, respectively. Qualitative data were analysed with Pearson's chi-squared test. A p-value $<0.05$ was considered statistically significant.

75 (43 males, 57\%) patients were recruited. 39 patients had a critical, 18 severe, and 18 mild-moderate disease [9]. Mean \pm SD time from discharge to outpatient visit was $97 \pm 26$ days. Seven (9\%) patients had a history of asthma, whereas no previous diagnosis of interstitial lung disease or COPD were reported. 26 (34\%) had a diagnosis of systemic hypertension, nine $(12 \%)$ of diabetes, three $(4 \%)$ of ischaemic heart disease, and three (4\%) of arrythmia. 43 (63\%) patients showed a residual parenchymal involvement at CT. Spirometry showed normal mean values: forced vital capacity was $104 \pm 17 \%$ predicted and $\mathrm{FEV}_{1} 100 \pm 16 \%$ predicted. However, mean $D_{\text {LCO }}$ was $71 \pm 14 \%$ of predicted with a mean haemoglobin level of $15.0 \pm 1.5$ $\mathrm{g} \cdot \mathrm{dL}^{-1}$. The average peak $V_{\mathrm{O}_{2}}^{\prime}$ of our population was $20.0 \mathrm{~mL} \cdot \mathrm{min}^{-1} \cdot \mathrm{kg}^{-1}$ corresponding to a mean $\pm \mathrm{SD}$ $83 \pm 15 \%$ of the predicted value; the mean \pm SD slope of the relation between ventilation and carbon dioxide output during exercise $\left(V_{\mathrm{E}}^{\prime} / V^{\prime} \mathrm{CO}_{2}\right.$ slope) was 28.4 \pm 3.1 , with median (interquartile range) peak exercise value for the alveolar-arterial gradient for oxygen of 26 (18-31) mmHg. 41 (55\%) patients showed a peak $V^{\prime} \mathrm{O}_{2}<85 \%$ of predicted (table 1 ). Patients with a reduced exercise capacity did not exhibit a ventilatory limitation by CPET (breathing reserve $<15 \%$ ), whereas 13 patients showed a circulatory limitation (HRR $<15 \%$ ), and 15 a reduced anaerobic threshold $(<45 \%)$ with or without reduction of HRR.

TABLE 1 Differences between patients with normal and reduced exercise capacity

\begin{tabular}{|c|c|c|c|}
\hline & Normal exercise capacity $(n=34)$ & Reduced exercise capacity $(n=41)$ & $\mathrm{p}$-value \\
\hline Male, n (\%) & $16(47)$ & $27(65)$ & 0.101 \\
\hline Age, years & $58 \pm 10$ & $56 \pm 13$ & 0.482 \\
\hline Smoking status never/current/ex-smoker, $\mathrm{n}(\%)$ & $21 / 4 / 9(62 / 12 / 26)$ & $28 / 10 / 3(68 / 24 / 8)$ & 0.700 \\
\hline $\mathrm{FEV}_{1}, \%$ pred & $107 \pm 19$ & $102 \pm 15$ & 0.170 \\
\hline FVC, \% pred & $103 \pm 18$ & $98 \pm 13$ & 0.215 \\
\hline Alveolar volume, \% pred & $89 \pm 13$ & $83 \pm 14$ & 0.063 \\
\hline CT abnormal/total, n (\%) & $19 / 30(63)$ & $24 / 41(58)$ & 0.683 \\
\hline CT-SS ${ }^{\natural}$ & $16.0 \pm 9.2$ & $18.6 \pm 10.7$ & 0.616 \\
\hline$\%$ V-RPI ${ }^{q}$ & $20(15-45)$ & $17(15-40)$ & 0.611 \\
\hline $\operatorname{mMRC}(0 / 1 / 2 / 3 / 4)$ & $15 / 13 / 6 / 0 / 0$ & $14 / 18 / 9 / 0 / 0$ & 0.672 \\
\hline$V_{\mathrm{O}_{2}}^{\prime}$ peak, \% pred & $97 \pm 9$ & $72 \pm 9$ & $<0.001$ \\
\hline Respiratory exchange ratio at peak & $1.18 \pm 0.09$ & $1.22 \pm 0.11$ & 0.121 \\
\hline Heart rate reserve, $\%$ & $10 \pm 11$ & $16 \pm 12$ & 0.040 \\
\hline Heart rate peak, bpm & $145 \pm 19$ & $138 \pm 22$ & 0.136 \\
\hline Oxygen pulse peak, $\%$ pred & $110 \pm 15$ & $85 \pm 19$ & $<0.001$ \\
\hline Ventilation peak, $\min ^{-1}$ & $67 \pm 21$ & $58 \pm 18$ & 0.068 \\
\hline$V_{\mathrm{E}}^{\prime} / V_{\mathrm{CO}_{2}}^{\prime}$ slope, $\mathrm{L} \cdot \mathrm{L}^{-1}$ & $28.1 \pm 3.2$ & $28.7 \pm 3.1$ & 0.453 \\
\hline$V_{\mathrm{E}}^{\prime} / V_{\mathrm{CO}_{2}}^{\prime}$ slope $>30, \mathrm{n}(\%)$ & $5(15)$ & $6(15)$ & 0.993 \\
\hline Alveolar-arterial gradient for $\mathrm{O}_{2}$ at peak ${ }^{+}, \mathrm{mmHg}$ & $26(19-31)$ & $26(16-31)$ & 0.719 \\
\hline$P_{\mathrm{aco}_{2}}$ at peak ${ }^{+}, \mathrm{mmHg}$ & $35 \pm 4$ & $35 \pm 4$ & 0.955 \\
\hline Lactate at peak ${ }^{+}, \mathrm{mmol} \cdot \mathrm{L}^{-1}$ & $7.5 \pm 2.7$ & $7.1 \pm 2.5$ & 0.464 \\
\hline Borg scale of dyspnoea at peak & $4.0 \pm 2.3$ & $3.5 \pm 2.3$ & 0.373 \\
\hline Borg scale of perceived exertion at peak & $5.3 \pm 2.0$ & $5.5 \pm 2.0$ & 0.638 \\
\hline \multicolumn{4}{|c|}{ 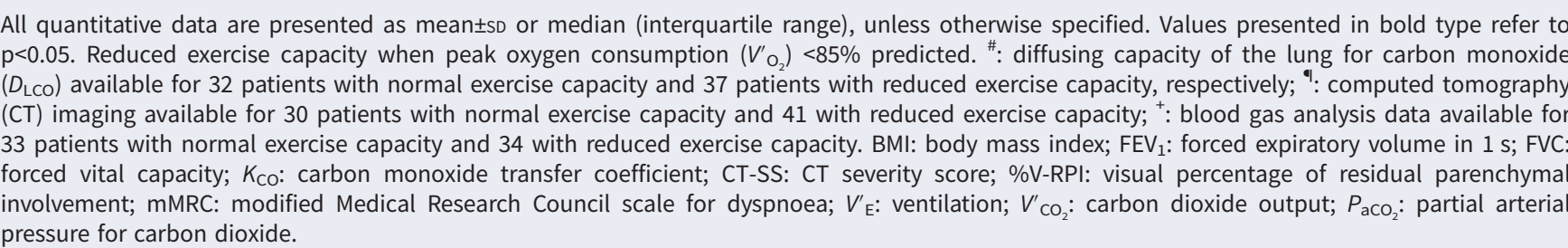 } \\
\hline
\end{tabular}


Patients with a reduced exercise capacity showed an early anaerobic threshold, indicating a higher degree of deconditioning; they reached lower levels of performance and earlier termination, with a lower work, a lower peak oxygen pulse, a higher HRR, and a wider breathing reserve. A reduced slope of oxygen uptake to work rate relationship ( $V_{\mathrm{O}_{2}}^{\prime} / \mathrm{WR}$ slope) in the exercise-limited subgroup is consistent with a worse anaerobic efficiency. Deconditioning might be related to a direct effect of the viral load on the muscle tissue, with an impaired $\mathrm{O}_{2}$ extraction and use [10], as well as to a prolonged hospital stay and post-hospitalisation syndrome. Remarkably, parameters of ventilatory efficiency or gas exchange were still in the limit of normal and we did not find a significant difference between patients with preserved and those with a reduced exercise capacity [11]; neither pulmonary function tests nor CT imaging helped to discriminate patients with a lower peak $V^{\prime}{ }_{\mathrm{O}_{2}}$. This is in line with the data reported by GAO et al. [12] on 10 COVID-19 survivors 1 month after discharge from rehabilitation. Nevertheless, RAman et al. [13] reported a reduced exercise capacity in a comparable proportion of moderate-to-severe COVID-19 survivors, although they showed a mild ventilatory inefficiency. No data on $D_{\text {LCO }}$ or gas exchange at peak of CPET were reported, but an explanation for this difference in residual ventilatory impairment could rely on the earlier evaluation time from discharge (median 1.6 months). Moreover, Ong et al. [14] showed that SARS survivors had only a mild reduction of lung function and exercise capacity at CPET, which could not be accounted for impairment of pulmonary function, with $41 \%$ presenting a reduced anaerobic threshold, in agreement with our findings.

In our study, symptoms at rest and at peak were comparable. Nevertheless, 39 (52\%) patients reported dyspnoea during their daily activity. Residual dyspnoea is frequently reported by COVID-19 survivors [15, 16]; its origin can depend on multiple factors, and a mildly impaired exercise capacity associated with deconditioning might play a role.

The main limitations of our study are its single-centre nature, which impacts on the generalisability, and the absence of a baseline assessment.

In conclusion, COVID-19 survivors show a mild reduction of their exercise capacity, probably caused by muscle deconditioning. This is the first study on CPET performance, pulmonary function tests and CT imaging, showing no relevant functional sequelae on ventilatory and gas exchange response to exercise. A longer follow-up is needed to evaluate the full spectrum of recovery.

\footnotetext{
Rocco Francesco Rinaldo $\odot^{1}$, Michele Mondoni ${ }^{1}$, Elena Maria Parazzini ${ }^{1}$, Federica Pitari $\oplus^{1}$, Elena Brambilla ${ }^{1}$, Simone Luraschi ${ }^{1}$, Maurizio Balbi ${ }^{2}$, Giuseppe Francesco Sferrazza Papa ${ }^{3,4}$, Giovanni Sotgiu $\oplus^{5}$, Marco Guazzi ${ }^{6}$, Fabiano Di Marco $\oplus^{7}$ and Stefano Centanni ${ }^{1}$
}

${ }^{1}$ Dept of Health Sciences, Respiratory Unit, ASST Santi Paolo e Carlo, San Paolo Hospital, University of Milan, Milan, Italy. ${ }^{2}$ Radiologic Sciences, Dept of Medicine and Surgery, University of Parma, Parma, Italy. ${ }^{3}$ Dipartimento di Scienze Neuroriabilitative, Casa di Cura del Policlinico, Milan, Italy. ${ }^{4}$ Dept of Health Sciences, University of Milan, Milan, Italy. ${ }^{5}$ Clinical Epidemiology and Medical Statistics Unit, Dept of Medical, Surgical and Experimental Medicine, University of Sassari, Sassari, Italy. ${ }^{6}$ Dept of Health Sciences, Cardiology Unit, ASST Santi Paolo e Carlo, San Paolo Hospital, University of Milan, Milan, Italy. ${ }^{7}$ Dept of Health Sciences, Respiratory Unit, ASST Papa Giovanni XXIII Hospital, University of Milan, Bergamo, Italy.

Corresponding author: Rocco Francesco Rinaldo (rocco.rinaldo@unimi.it)

Acknowledgements: The authors wish to acknowledge Silvia Terraneo, Fausta Alfano, Andrea Baccelli, Matteo Davì, Sabrina De Pascalis, Alessandra Masseroni, Stefano Pavesi and Silvia Ruggeri for their help in patient recruitment and data collection, and their work in the post-COVID-19 outpatient service. Authors also wish to thank our outstanding CPET Laboratory nurses Giulia Merli, Claudia Migliaccio and Caterina Spagnuolo.

Conflict of interest: None declared.

Support statement: This work was funded by Università degli Studi di Milano in the context of the Registry for COVID19 Emergency (RECOVER) electronic database. Funding information for this article has been deposited with the Crossref Funder Registry. 
References

1 Mondoni M, Sferrazza Papa GF, Rinaldo R, et al. Utility and safety of bronchoscopy during the SARS-CoV-2 outbreak in Italy: a retrospective, multicentre study. Eur Respir J 2020; 56: 2002767.

2 Bai C, Chotirmall SH, Rello J, et al. Updated guidance on the management of COVID-19: from an American Thoracic Society/European Respiratory Society coordinated International Task Force (29 July 2020). Eur Respir Rev 2020; 29: 200287.

3 Wang W, Xu Y, Gao R, et al. Detection of SARS-CoV-2 in different types of clinical specimens. JAMA 2020; 323: 1843-1844.

4 Weisman IM, Marciniuk D, Martinez FJ, et al. ATS/ACCP statement on cardiopulmonary exercise testing. Am J Respir Crit Care Med 2003; 167: 211-277.

5 Rinaldo RF, Mondoni M, Comandini S, et al. The role of phenotype on ventilation and exercise capacity in patients affected by COPD: a retrospective study. Multidiscip Respir Med 2020; 15: 13-19.

6 Yang R, Li X, Liu H, et al. Chest CT Severity Score: an imaging tool for assessing severe COVID-19. Radiol Cardiothorac Imaging 2020; 2: e200047.

7 Colombi D, Bodini FC, Petrini M, et al. Well-aerated lung on admitting chest CT to predict adverse outcome in COVID-19 pneumonia. Radiology 2020; 296: E86-E96.

8 Huang $\mathrm{Y}$, Tan C, Wu J, et al. Impact of coronavirus disease 2019 on pulmonary function in early convalescence phase. Respir Res 2020; 21: 163.

9 World Health Organization. Clinical Management of COVID-19: Interim Guidance, 27 May 2020. Geneva, WHO, 2020. Available from: https://apps.who.int/iris/handle/10665/332196

10 Kucuk A, Cumhur Cure M, Cure E. Can COVID-19 cause myalgia with a completely different mechanism? A hypothesis. Clin Rheumatol 2020; 39: 2103-2104.

11 Hansen JE, Sue DY, Wasserman K. Predicted values for clinical exercise testing. Am Rev Respir Dis 1984; 129: S49-S55.

12 Gao Y, Chen R, Geng Q, et al. Cardiopulmonary exercise testing might be helpful for interpretation of impaired pulmonary function in recovered COVID-19 patients. Eur Respir J 2021; 57: 2004265.

13 Raman B, Cassar MP, Tunnicliffe EM, et al. Medium-term effects of SARS-CoV-2 infection on multiple vital organs, exercise capacity, cognition, quality of life and mental health, post-hospital discharge. EClinicalMedicine 2021; 31: 100683.

14 Ong KC, Ng AWK, Lee LSU, et al. Pulmonary function and exercise capacity in survivors of severe acute respiratory syndrome. Eur Respir J 2004; 24: 436-442.

15 Sonnweber T, Sahanic S, Pizzini A, et al. Cardiopulmonary recovery after COVID-19: an observational prospective multicentre trial. Eur Respir J 2021; 57: 2003481.

16 Lerum TV, Aaløkken TM, Brønstad E, et al. Dyspnoea, lung function and CT findings 3 months after hospital admission for COVID-19. Eur Respir J 2021; 57: 2003448. 